Chronic Obstructive Pulmonary Diseases: Journal of the COPD Foundation

\title{
Non-Invasive Assessment and Management of Liver Involvement in Adults With Alpha-1 Antitrypsin Deficiency
}

\author{
Karim Hamesch, $\mathrm{MD}^{1,2}$ Pavel Strnad, $\mathrm{MD}^{1,2}$
}

\begin{abstract}
Alpha-1 antitrypsin deficiency (AATD) is a systemic disorder affecting mainly the lung and the liver and is caused by mutations in SERPINA 1, the AAT gene. A homozygous " $\mathrm{Pi}{ }^{*} \mathrm{Z}$ " mutation ( $\mathrm{Pi}{ }^{*} \mathrm{ZZ}$ genotype) may cause liver fibrosis on its own independently of pulmonary AATD manifestation, while heterozygous $\mathrm{Pi}^{*} \mathrm{Z}$ carriage ( $\mathrm{Pi}^{*} \mathrm{MZ}$ genotype) is considered a strong risk factor for development of liver cirrhosis in patients with concomitant liver disease such as alcoholic and non-alcoholic liver disease. In $\mathrm{Pi}^{*} \mathrm{ZZ}$ homozygotes, liver disease constitutes the second leading cause of death and is highly heterogeneous. About $35 \%$ of $\mathrm{Pi}^{*} \mathrm{ZZ}$ individuals display significant liver fibrosis on biopsy (i.e., fibrosis stage $\geq 2$ on scale $0-4$ ). Among non-invasive methods for liver fibrosis assessment, liver stiffness measurement (LSM) via vibration-controlled transient elastography (VCTE) has been most widely evaluated. Based on these data, $\mathrm{Pi}^{*} \mathrm{ZZ}$ adults have $20 \mathrm{x}$ increased odds of developing advanced liver fibrosis (i.e., fibrosis stage $\geq 3$ ) than adults without AAT mutation. Risk factors for accelerated fibrosis progression are male sex, age $\geq 50$ years, alcohol misuse, obesity, diabetes mellitus, or metabolic syndrome. Unlike VCTE, other ultrasound- and magnetic resonance-based elastography methods have been assessed in small cohorts of $\mathrm{Pi}^{*} \mathrm{ZZ}$ individuals and remain to be comprehensively validated. Among blood-based fibrosis tests, AST-to-platelet ratio index (APRI) correlates moderately with histologic fibrosis stage and LSM. Given APRI's wide availability, it can be used for risk stratification as an adjunct to LSM or when LSM is not at hand. Despite recent efforts, AATD-related liver disease, especially for genotypes other than $\mathrm{Pi}^{*} \mathrm{ZZ}$, remains greatly understudied. AATD individuals should be offered liver biochemistry, liver ultrasound, and non-invasive fibrosis assessment at the time of diagnosis to detect potential complications and for proper risk stratification. If signs of AATD-related liver disease occur (i.e., pathologic fibrosis test or repeatedly elevated liver enzymes), patients should be referred to a health care center specialized in AATD-related liver disease and be screened for potentially treatable comorbidities. To exclude the latter, they may need a liver biopsy. Moreover, every health care provider of an AATD individual should be aware of the potential liver manifestation, counsel their patient on modifiable hepatic risk factors, and offer them regular liver check-ups.
\end{abstract}

\footnotetext{
Abbreviations: alpha-1 antitrypsin deficiency, AATD; liver stiffness measurement, LSM; vibration controlled transient elastography, VCTE; AST-to-platelet ratio, APRI; fibrosis-4, FIB4; enhanced liver fibrosis, ELF; non-alcoholic fatty liver disease, NAFLD; body mass index, BMI; acoustic radiation force impulse imaging, ARFI; 2D-shear wave elastography, 2D-SWE; controlled attenuation parameter, CAP; magneticresonance-based elastography, MRE; proton density fat fraction, PDFF; area under the receiver operating curve, AUROC; gamma-glutamyl transferase, GGT; hepatocellular carcinoma, HCC; alcohol fatty liver disease, ALD

Funding Support: This work was supported by the EASL registry grant on alpha-1 antitrypsin-related liver disease, the Deutsche Forschungsgemeinschaft (DFG) consortium SFB/TRR57 "Liver fibrosis", the Interdisciplinary Center for Clinical Research (IZKF) within the medical faculty at RWTH Aachen University, the Else Kroener Excellence Fellowship, (all to P.S.) as well as the START program within the medical faculty at RWTH Aachen University, the German Liver Foundation, and the German Gastroenterological Association (all to K.H.).

Date of Acceptance: February 13, 2020

Citation: Hamesch K, Strnad P. Non-invasive assessment and management of liver involvement in adults with alpha-1 antitrypsin deficiency. Chronic Obstr Pulm Dis. 2020;7(3):260-271. doi: https://doi.org/10.15326/jcopdf.7.3.2019.0161
} 
1 Medical Clinic III, Gastroenterology, Metabolic Diseases and Intensive Care, University Hospital RWTH Aachen, Aachen, Germany

2 Coordinating Center for Alpha-1 Antitrypsin DeficiencyRelated Liver Disease of the European Reference Network on Hepatological Diseases (ERN RARE-LIVER) and the European Association for the Study of the Liver (EASL) Registry Group "Alpha-1 Liver"

\section{Address correspondence to:}

Pavel Strnad, MD

European Reference Network on Hepatological Diseases

European Association for the Study of the Liver Registry Group University Hospital Aachen

Aachen, Germany

Email: pstrnad@ukaachen.de

Phone: +49 241 80-35324

\section{Keywords:}

liver fibrosis; liver stiffness; elastography; SERPINA 1; genetic liver disease; rare liver disease; alpha-1 antitrypsin deficiency; AATD

\section{What is Liver Fibrosis and What Are Its Consequences?}

Chronic liver disease of any etiology may result in liver scarring (liver fibrosis) as the uniform response to ongoing liver damage. Cirrhosis is the end-stage of such a process. ${ }^{1}$ Mechanistically, the persistent hepatic insult leads to loss of hepatocytes and release of proinflammatory mediators that result in chronic inflammation and activation of hepatic stellate cells. ${ }^{2}$ The latter are transformed into myofibroblasts and represent major producers of extracellular matrix. ${ }^{2}$ Importantly, the presence of liver fibrosis, but not other histological features such as liver steatosis (fatty liver) or chronic inflammation, is associated with increased hepatic morbidity and mortality., 3 Because of that, a proper assessment of liver fibrosis is essential to understand the severity of liver disease as well as the prognosis in order to guide further clinical management. ${ }^{5}$

Patients with chronic hepatitis or early liver fibrosis are usually asymptomatic or present with unspecific symptoms, hence, they typically remain undiagnosed for many years. This is facilitated by the fact, that detailed medical history and physical examination are often not sufficient for detecting advanced liver fibrosis. ${ }^{6}$ Indeed, patients with fibrosis or even cirrhosis may present with normal serum liver enzymes. ${ }^{6}$ While patients with early stages of so-called compensated cirrhosis might still be asymptomatic, their decompensation is of great clinical relevance since it may lead to multiple life-threatening sequelae. Notably, the diagnosis of compensated cirrhosis results in a $4.7 x$ increased risk of death compared to the general population while decompensated cirrhosis is associated with a nearly $10 \mathrm{x}$ higher risk. ${ }^{7}$ The average life expectancy of patients with compensated cirrhosis is 10 to 13 years, but drops to 2 years when a decompensation occurs. ${ }^{8}$ As a consequence, cirrhosis is the 13 th leading cause of death globally. ${ }^{1}$ Moreover, hepatocellular carcinoma, which almost exclusively occurs in the cirrhotic liver, is the 4th leading cause of cancer-related death and its incidence continues to grow. $^{9}$

The main complications seen in individuals with decompensated cirrhosis are typically related to an elevated blood pressure in the portal venous system. ${ }^{1,10}$ This can result in ascites, esophageal varices, hepatorenal syndrome, and/or hepatic encephalopathy. While ascites may be refractory and greatly restrict life quality, spontaneous bacterial peritonitis may be fatal due to progression to multi-organ failure if not treated early. Esophageal varices may result in severe bleeding and hepatorenal syndrome in persistent renal failure, both reducing life expectancy. Finally, hepatic encephalopathy may be highly debilitating. Additional organs such as the lungs (portopulmonary hypertension or hepatopulmonary syndrome) or the heart (cirrhotic cardiomyopathy) can also become affected due to cirrhosis and the resulting alterations.

The presented diagnostic challenges as well as the deadly consequences of cirrhosis, especially if diagnosed at the late stage when a patient becomes symptomatic, highlight the importance of detecting liver fibrosis as early as possible to prevent further progression and/or to discuss the possibility of liver transplantation. This is particularly true in conditions known to associate with liver involvement such as alpha-1 antitrypsin deficiency.

\section{How to Assess Liver Fibrosis?}

For decades, liver biopsy was the key diagnostic procedure in hepatology and was used to evaluate the cause and stage of liver disease. Consequently, it was performed to facilitate the treatment decisions and to determine the prognosis. ${ }^{5}$ While biopsy is still considered the gold standard, the clinical approach to patients with chronic liver disease has undergone 
a transformation with an increased use of noninvasive tools to assess the extent of liver fibrosis. ${ }^{5}$ Notwithstanding its importance to assess the nature of a distinct liver disease, ${ }^{11}$ liver biopsy has several disadvantages that limit its use as a first-line tool to assess the stage of fibrosis. A major concern is its invasiveness with complications such as pain, bleeding, injury to the bile ducts, infection, or rarely, even death. ${ }^{5}$ Other important issues are the so-called sampling bias arising from the fact that the disease is often not uniformly distributed throughout the liver ${ }^{12-14}$ and an observer bias caused by the subjective assessment of pathologists regarding both severity and etiology of disease. ${ }^{12}$ Additionally, sampling size is not well standardized and may greatly affect the pathologist's interpretation. ${ }^{15}$ Therefore, a single biopsy might have limited predictive value ${ }^{14}$ and the necessity to perform serial biopsies to confirm the etiology and to monitor the course of disease further potentiates the risk of complications. Because of all of these issues, liver biopsy constitutes a significant burden for the health care system (i.e., the need of an expert gastroenterologist [or radiologist] and pathologist as well as the required periprocedural monitoring) and the patient (i.e., inability to work for a significant amount time).

In the past 3 decades, several non-invasive tools for assessing liver fibrosis have been developed and validated for use in the clinical routine. While these methods typically cannot accurately differentiate the stages of fibrosis, they are useful in ruling-out the presence of clinically significant liver fibrosis (i.e., fibrosis stage of 2 or higher on a $0-4$ scale) and in ruling-in the presence of advanced liver fibrosis (i.e., fibrosis stage of 3 or 4). ${ }^{16}$ From the less to the more technically complicated alternatives, these non-invasive fibrosis tests include: blood-based tests, ultrasound-based elastography, and magneticresonance-based elastography (Table 1). ${ }^{5,17}$

Blood tests include the ubiquitously available indirect tests, that are based on routine laboratory parameters (e.g., AST-to-platelet ratio index[APRI]) and fibrosis-4 [FIB4]) as well as the direct tests, using more sophisticated parameters and protected formulas (e.g., enhanced liver fibrosis [ELF $]^{\circledR}, \mathrm{HepaScore}^{\circledR}$, and FibroTest $\left.{ }^{\circledR}\right) .{ }^{18}$ The indirect blood tests generally reflect the amount of liver injury and/or the alterations occurring during progressive liver scaring and thereby mirror the extent of disease. For instance, thrombocytopenia is regarded as the earliest indicator of cirrhosis among routine blood tests, capturing multiple processes such as diminished liver function (reduced thrombopoietin production) and portal hypertension (splenic blood sequestration). 5,6,19 However, some tests depend on the etiology of chronic liver disease such as the "non-alcoholic liver disease (NAFLD) fibrosis score" that includes the body mass index (BMI) and the presence of diabetes mellitus. Direct blood tests try to circumvent the low specificity of parameters employed in indirect test scores and instead of that, take advantage of parameters of fibrogenesis and/or fibrinolysis. However, even this more direct approach may lead to false positive/ negative results. Moreover, these assays are less widely available and costlier than indirect tests. ${ }^{5,18}$

Since conventional liver imaging fails to reliably detect early stages of liver fibrosis, elastographic methods measuring the elasticity of the liver tissue emerged as tools to fill this diagnostic gap. These take advantage of a shear wave, that is sent from the probe through the chest wall into the liver. Subsequently, the velocity of wave propagation is evaluated by a receiver in the probe. Given that the velocity is related to the elasticity of the tissues, it is converted into a measurement of liver stiffness. ${ }^{5,17}$ In most entities of chronic liver disease, liver stiffness robustly correlates with the extent of liver fibrosis. ${ }^{5,17}$ Likely because of that, liver stiffness is incrementally predictive of adverse outcomes of chronic liver disease. ${ }^{5,20,21}$ For the various entities, specific liver stiffness cut-offs for ruling-out significant liver fibrosis and ruling-in cirrhosis have been proposed. ${ }^{17}$ However, the values of liver stiffness (i.e., $\mathrm{kPa}$ or $\mathrm{m} / \mathrm{s}$ ) determined by different elastographic tools are not interchangeable. Hence, cut-offs have still to be seen in the context of the underlying etiology and the used method and still carry a certain degree of uncertainty. However, as elastography is increasingly used as a first line tool to assess the extent of liver fibrosis, some authors proposed etiology-unspecific cut-offs. ${ }^{17}$

When used properly, elastography is excellent in ruling-out significant liver fibrosis (i.e., excellent negative likelihood ratios for $F \leq 1$ ) and rulingin advanced liver fibrosis (i.e., excellent positive likelihood ratios for $\mathrm{F} \geq 3$ ). However, several potential confounders must be considered when elevated liver stiffness values are measured to make sure that these really reflect an advanced scaring. Among others, 


\section{Table 1. Comparison of the Commonly Used Methods for Assessment of Liver Fibrosis}

\begin{tabular}{|c|c|c|c|c|c|}
\hline Parameter & Liver Biopsy & $\begin{array}{c}\text { Indirect } \\
\text { Blood Tests }\end{array}$ & $\begin{array}{c}\text { Direct } \\
\text { Blood Tests }\end{array}$ & $\begin{array}{l}\text { US-Based } \\
\text { Elastography }\end{array}$ & $\begin{array}{l}\text { MR-Based } \\
\text { Elastography }\end{array}$ \\
\hline Examples & $\begin{array}{r}\text { Percutaneous, } \\
\text { Transjugular, } \\
\text { Mini-laparoscopic }\end{array}$ & $\begin{array}{l}\text { APRI, } \\
\text { FIB4, } \\
\text { NFS }\end{array}$ & $\begin{array}{r}\text { FibroTest, } \\
\text { HepaScore, } \\
\text { ELF }\end{array}$ & $\begin{array}{r}\text { VCTE, ARFI, 2D-SWE } \\
\text { (different manufacturers) }\end{array}$ & (different manufacturers) \\
\hline Accuracy & ++ to +++ & + to ++ & ++ & ++ & ++ to +++ \\
\hline Validation & +++ (Gold Std) & ++ & ++ & ++ to +++ & ++ to +++ \\
\hline Safety & - & +++ & +++ & +++ & +++ \\
\hline Availability & ++ & +++ & + & ++ & + \\
\hline Cost & +++ & - to + & + to ++ & ++ & +++ \\
\hline Monitoring & + & +++ & + & ++ & + \\
\hline
\end{tabular}

- = negative; $+/++/+++=$ positive (in increasing degrees)

US=ultrasound; MR=magnetic resonance; APRI=AST-to-platelet ratio index; FIB4=fibrosis-4 index; NFS=non-alcoholic fatty liver diseases fibrosis score; $E L F=$ enhanced liver fibrosis test; VCTE=vibration-controlled transient elastography (FibroScan); ARFI=acoustic radiation force impulse imaging; 2D-SWE=2D shear wave elastography; std=standard

non-fasting (i.e., the postprandially increased blood flow through the liver), severe inflammation (i.e., liver enzymes $>5 \mathrm{x}$ the upper limit of normal), or passive liver congestion (i.e., heart failure) may result in high stiffness values that potentially overestimate the amount of liver fibrosis. $5,17,22$

The most widely available ultrasound-based elastography methods are: vibration-controlled transient elastography (VCTE; FibroScan ${ }^{\circledR}$; Echosens, France), acoustic radiation force impulse imaging (ARFI; Siemens, Germany), and 2D-shear wave elastography (2D-SWE; offered by different companies [e.g. ,Toshiba, Japan]). ${ }^{17}$ All methods are used to measure liver stiffness of the right liver lobe and the measurement is performed via an intercostal access. Of these methods, VCTE is the most widely used one and is backed up by the largest amount of scientific evidence. ${ }^{5,17,23}$ In addition, VCTE offers a simultaneous measurement of controlled attenuation parameter (CAP), that is used as a surrogate of hepatic steatosis. $^{24}$ On the other hand, ARFI and 2D-SWE allow a simultaneous visualization of the area in which liver stiffness is measured, whereas VTCE is performed in a "blind" manner. ${ }^{17}$

Magnetic-resonance-based elastography (MRE) is another attractive method for assessment of liver stiffness. It is regarded to be at least equivalent to VCTE in distinguishing between advanced and nonadvanced liver fibrosis. ${ }^{25,26}$ Similar to VCTE, it can also quantify hepatic steatosis using proton density fat fraction (PDFF). Notably, MRE appears to perform better than VCTE in patients with severe obesity. ${ }^{5}$ Another advantage of MRE is the fact that it analyzes the liver as a whole while all ultrasound-based elastography methods assess only a limited region of the liver. Still, basically the same confounders of liver stiffness measurement apply to MRE as to the ultrasound-based techniques. In conclusion, MRE is equal or possibly even superior to VCTE, especially in difficult cases, but is less widely available and remains to be standardized between health care centers and different vendors. ${ }^{27}$

As non-invasive risk stratification is emerging as the new standard of initial liver disease evaluation, risk assessment has shifted from histological fibrosis staging to the dichotomization of fibrosis as advanced versus non-advanced. ${ }^{3-5}$ Thus, the current strategy is to triage the patients into different risk categories. Consequently, liver biopsy is employed for individuals with inconclusive non-invasive results as well as for obtaining additional diagnostic clues. This approach greatly reduced the number of performed liver biopsies. A commonly used strategy is to use an indirect bloodbased test (e.g., APRI or FIB-4) and an elastographic method (e.g., VCTE). ${ }^{5}$ If both tests concordantly indicate that advanced liver fibrosis is not present, a more or less stringent follow-up is recommended. If both tests concordantly indicate the presence of advanced liver fibrosis, the patient should be managed accordingly (e.g., monitoring for complications of cirrhosis). If both tests are discordant (and if potential confounders of both blood-based and elastography- 
based tests had previously been excluded), liver biopsy should be discussed, especially if it affects further management.

Taken together, non-invasive measures of liver fibrosis assessment are increasingly available, have no/minimal procedure-related risks and are less costly than biopsy. Therefore, they are well suited for a highthroughput monitoring of liver disease progression. This is particularly relevant given the high burden of NAFLD affecting up to $30 \%$ of the population. Despite the reduced need for liver biopsy, histology remains an important tool in specific indications, such as in individuals with an inconclusive result of non-invasive assessment or in patients, where further information about the etiology and/or activity of liver injury are needed.

\section{What is the Liver Disease Burden in Adults With the $\mathrm{Pi}^{*} \mathrm{ZZ}$ and $\mathrm{Pi}^{*} \mathrm{MZ}$ Genotype of Alpha-1 Antitrypsin Deficiency?}

Alpha-1 antitrypsin deficiency (AATD) is one of the most common, potentially lethal genetic diseases worldwide. While over 100 mutations of the SERPINA 1 gene are known, the " $\mathrm{Pi}^{*} \mathrm{Z}$ " variant (Glu342Lys) is the most relevant mutation. Heterozygous $\mathrm{Pi}^{*} \mathrm{Z}$ carriage is termed as " $\mathrm{Pi}^{*} \mathrm{MZ}$ " genotype and occurs in about 1:30 whites. Homozygosity of the $\mathrm{Pi}^{*} \mathrm{Z}$ variant is termed as the " $\mathrm{P}{ }^{*} \mathrm{ZZ}$ " genotype and is seen in about 1:3000 whites. AATD is a multi-systemic disease mainly affecting the lungs (due to deficiency of AAT in the circulation) and the liver (due to intrahepatic accumulation of misfolded AAT) ${ }^{28-30}$ While AATDrelated lung disease is the most common disease manifestation, liver disease is the second most common organ involvement. It displays a biphasic pattern in that it typically occurs either in early childhood or late adulthood. ${ }^{31}$ Notably, the relative proportion of AATD-related liver disease is higher in never-smokers compared to smokers. ${ }^{32,33}$ An increasing prevalence of AATD-related liver disease is to be expected due to: (1) the decreasing proportion of smokers in the population and consequently a decreasing proportion of AATD-related lung disease; (2) the current epidemic of obesity that constitutes an important co-factor in liver disease development; ${ }^{34,35}$ (3) the increased awareness of AATD-related liver disease among patients and physicians; and (4) the growing number of therapeutic studies targeting the liver. ${ }^{36}$

While diagnosis of adult liver disease in general is difficult, early diagnosis of liver involvement in patients with AATD - whether AATD itself is diagnosed or not - is particularly challenging. ${ }^{18} \mathrm{~A}$ major reason is that in homozygous $\mathrm{Pi}^{*} \mathrm{ZZ}$ adults, liver enzymes are frequently not elevated despite significant liver fibrosis. ${ }^{37,38}$ Additionally, likely due to low awareness about the importance of liver disease burden, liver enzymes are not determined regularly. ${ }^{38}$ To change that, several recent studies evaluated the liver phenotype of $\mathrm{Pi}^{*} \mathrm{ZZ}$ individuals. First, a biopsy study evaluated the amount of liver fibrosis in 94 noncirrhotic $\mathrm{Pi}^{*} \mathrm{ZZ}$ individuals and observed significant liver fibrosis in $35 \%$ of them. ${ }^{39}$ This endeavor also validated the usefulness of several blood- and elastography-based fibrosis scores to predict the extent of histological liver fibrosis. ${ }^{39}$ APRI and FIB-4 both predicted the presence of clinically significant fibrosis on liver histology (i.e., fibrosis stage of at least 2 on a 0-4 METAVIR scale). The areas under the receiver operating curve (AUROC) were 0.69 and 0.66 for APRI and FIB-4, respectively. VCTE was also validated in this cohort and had an AUROC of 0.70 for significant liver fibrosis. Interestingly, the gamma-glutamyl transferase (GGT) test was numerically better for recognizing the presence of significant fibrosis (i.e., $F \geq 2$ ) with an AUROC of 0.77 compared to indirect blood tests as well as VCTE. However, VCTE was better than all blood-based parameters to distinguish advanced liver fibrosis (i.e., $\mathrm{F} \geq 3$ compared to $\mathrm{F} \leq 2$; AUROC 0.92) and this finding was confirmed in an independent study assessing $\mathrm{P}^{*} \mathrm{ZZ}$ adults with advanced liver fibrosis. ${ }^{40}$ This is reminiscent of the situation in other chronic liver diseases as liver stiffness is known to be useful to detect advanced liver fibrosis but is less well suited to demarcate mild or significant liver fibrosis stages (i.e., $\mathrm{F} \leq 2){ }^{17}$

VCTE was also used in a large multinational cohort comprising $554 \mathrm{Pi}^{*} \mathrm{ZZ}$ adults and proved to be an easy-to-use tool which was cross-validated against the aforementioned APRI score (indirect blood test) as well as the patented HepaScore (direct blood test). This multinational effort confirmed the high prevalence of significant liver fibrosis in $\mathrm{Pi}^{*} \mathrm{ZZ}$ individuals. Depending on the method, significant fibrosis was seen in $20 \%-36 \%$ of them and advanced liver fibrosis was 9-20x more frequent in $\mathrm{Pi}^{*} \mathrm{ZZ}$ individuals compared to individuals without AAT mutation. ${ }^{38}$ These data are 
in line with other observations. In particular, $\mathrm{Pi}^{*} \mathrm{ZZ}$ individuals are about $20 \mathrm{x}$ more likely to require liver transplantation than non-carriers (Table 2). ${ }^{38,41,42}$

The above described, VCTE-based study ${ }^{38}$ offers an unprecedented insight into a large population of $\mathrm{Pi}^{*} \mathrm{ZZ}$ individuals and allows an assessment of disease modifying factors. This is particularly important, since $\mathrm{Pi}^{*}$ ZZ-related liver disease is highly heterogeneous. ${ }^{43}$ This heterogeneity is likely due to both additional genetic factors as well as second hits, that presumably result in accelerated liver fibrogenesis. While these modifiers remain largely unknown, male sex, age $\geq$ 50 years, obesity, and presence of diabetes and/or metabolic syndrome play an important role. $38,39,43,44$ While alcohol misuse - as one of the leading causes of liver disease worldwide - was excluded from studies with $\mathrm{Pi}^{*} \mathrm{ZZ}$ adults, it is likely that alcohol misuse is also a relevant risk factor for liver fibrogenesis. This is also supported by the strong impact of heterozygous $\mathrm{Pi}^{*} \mathrm{Z}$ carriage on cirrhosis development in adults with alcohol misuse. ${ }^{45}$ Notably, the above described parameters have all been associated with accelerated liver disease progression in other liver disorders. Importantly, the severity of lung and liver disease do not seem to correlate, which is well in line with the fact that they are pathomechanistically different. ${ }^{38}$

Several smaller studies tested other modalities of non-invasive liver fibrosis assessment in $\mathrm{Pi}^{*} \mathrm{ZZ}$ adults: 2 studies employed $\mathrm{MRE}^{46,47}$ with one of them validating its usefulness in a small cohort of 11 biopsied $\mathrm{Pi}^{*} \mathrm{ZZ}$ adults. ${ }^{43}$ This very limited evidence suggests that MRE might be suitable to exclude the presence of significant liver fibrosis. However, the current data need to be confirmed in larger investigations. While MRE has the advantage of assessing the whole liver with high accuracy, its main disadvantage is the low availability and the high costs which limits its applicability for the routine evaluation of $\mathrm{Pi}^{*} \mathrm{ZZ}$ individuals.

Two other studies evaluated other ultrasound-based elastography methods (ARFI ${ }^{47,48}$ and $2 \mathrm{D}-\mathrm{SWE}^{47}$ ). However, ARFI and 2D-SWE approaches were not validated against liver histology and these methods are, even for other disease entities, not as wellvalidated as VCTE. ${ }^{17}$ Notwithstanding this limitation, in a small $\mathrm{Pi}^{*} \mathrm{ZZ}$ cohort, ARFI, 2D-SWE, and MRE correlated strongly with each other. ${ }^{47}$ Therefore, ARFI, and 2D-SWE might be useful in conjunction with a blood-based fibrosis score to assess whether advanced liver fibrosis is present. As for other liver diseases, ${ }^{5}$ the

\section{Table 2. Comparison of Liver-related Risks in Adults With the Homozygous Pi*ZZ and Heterozygous Pi*MZ Genotype}

\begin{tabular}{|c|c|c|}
\hline Parameter & $\mathrm{Pi}$ ZZZ adults & Pi*MZ adults \\
\hline Prevalence & $1: 2000-1: 4000$ & $1: 25-1: 50$ \\
\hline Impact on Liver Disease & $\begin{array}{r}\text { Disease-causing } \\
\text { Significant: } 20 \%-36 \%\end{array}$ & $\begin{array}{r}\text { Disease-modifying, } \\
\text { potentially disease-causing(?) }\end{array}$ \\
\hline $\begin{array}{l}\text { Prevalence of Liver Fibrosis } \\
\text { in Absence of Concomitant } \\
\text { Liver Disease }\end{array}$ & $\begin{array}{r}\text { Advanced: } 5 \%-26 \% \text {; } 9-20 x \text { Odds (adjusted } \\
\text { for age, sex, BMI, diabetes, and alcohol } \\
\text { consumption) }\end{array}$ & unknown \\
\hline Cirrhosis Risk & Absolute risk up to $10 \%$ & $\begin{array}{r}3-7 \mathrm{x} \text { odds } \\
\text { (adjusted; in alcohol misuse, } \\
\text { NAFLD, and cystic fibrosis: 6-7x) }\end{array}$ \\
\hline $\begin{array}{l}\text { Hepatic Decompensation Risk } \\
\text { in Cirrhotics }\end{array}$ & Not known & $\begin{array}{l}\text { More common (at least ascites and } \\
\text { hepatic encephalopathy and } 2.5 x \\
\text { odds of having significant portal } \\
\text { hypertension) }\end{array}$ \\
\hline Liver Transplantation Risk & $20 x$ & $3-4 \mathrm{x}(\sim 10 \%$ of transplant recipients) \\
\hline HCC De Novo Risk & Not known & Likely not increased \\
\hline $\begin{array}{l}\text { Risk Factors for Liver } \\
\text { Fibrogenesis }\end{array}$ & $\begin{array}{r}\text { Established: Male sex, age } \geq 50 \text { years, } \\
\text { obesity, metabolic syndrome } \\
\text { Likely: alcohol misuse }\end{array}$ & $\begin{array}{l}\text { Established: Alcohol misuse, obesity/ } \\
\text { metabolic syndrome Likely: Male } \\
\text { sex, age } \geq 50 \text { years }\end{array}$ \\
\hline
\end{tabular}

$\mathrm{HCC}=$ hepatocellular carcinoma 
combination of a blood-based fibrosis test (with APRI being the most validated one) and an elastography method (with VCTE being the most established one) offers the best, currently available diagnostic accuracy and inconclusive results should foster liver biopsy. A combination of different methods might be especially helpful for patients with intermediate liver stiffness values (i.e., $5-7 \mathrm{kPa}$ ).

In addition to liver fibrosis assessment, the recently published studies revealed other interesting features of $\mathrm{Pi}^{*} \mathrm{ZZ}$-related liver disease. For example, 44\% of $\mathrm{Pi}^{*} \mathrm{ZZ}$ individuals displayed histological liver steatosis ${ }^{39}$ and these data meshed well with noninvasive evaluations using CAP as a surrogate of liver steatosis. ${ }^{24}$ In particular, CAP $\geq 280 \mathrm{~dB} / \mathrm{m}$, suggesting severe steatosis (grade 3 ), was detected in $39 \%$ of $\mathrm{Pi}^{*} \mathrm{ZZ}$ homozygotes versus $31 \%$ of non-carriers while $C A P \geq 248 \mathrm{~dB} / \mathrm{m}$, suggesting the presence of steatosis grade $\geq 1$, was present in $61 \%$ of $\mathrm{Pi}^{*} \mathrm{ZZ}$ individuals. ${ }^{38}$ Another small study using CAP detected steatosis grade $\geq 1$ in $65 \%$ of $\mathrm{Pi}^{*} \mathrm{ZZ} / \mathrm{Pi}^{*} \mathrm{SZ}$ adults and grade $\geq$ 2 in $52 \%$ of individuals. ${ }^{49}$ However, the accuracy of CAP for predicting histological steatosis has not been histologically validated in AATD yet. As a potential underlying mechanism, $\mathrm{Pi}^{*} \mathrm{ZZ}$ individuals displayed lower serum triglyceride, VLDL, and LDL cholesterol concentrations than $\mathrm{Pi}^{*} \mathrm{Z}$ non-carriers which points to the hypothesis that $\mathrm{Pi}^{*} \mathrm{ZZ}$ individuals may have impaired hepatic lipid secretion. These findings were supported by the observations in transgenic mice overexpressing the $\mathrm{Pi}^{*} \mathrm{Z}$ mutation, that also harboured mild liver steatosis. ${ }^{38}$

While the above described studies allowed a solid insight into the likelihood of $\mathrm{Pi}{ }^{*} \mathrm{ZZ}$ individuals to suffer from cirrhosis, their risk to develop hepatocellular carcinoma (HCC) has not been systematically analyzed. While early studies suggested a high risk, several others imply a lower likelihood and suggest that HCC develops almost exclusively in cirrhotic $\mathrm{Pi}^{*} \mathrm{ZZ}$ individuals. ${ }^{50,51}$ Therefore, additional studies are needed to clarify this discrepancy. Likewise, longitudinal studies assessing the exact risk of hepatic decompensation in $\mathrm{Pi}^{*} \mathrm{ZZ}$ adults need to be performed. These studies are crucial for development of a hepatic surveillance plan.

In contrast to the $\mathrm{Pi}^{*} \mathrm{ZZ}$ genotype that is diseasecausing and therefore may lead to cirrhosis development on its own, heterozygous $\mathrm{Pi}^{*} \mathrm{Z}$ carriage ( $\mathrm{Pi}^{*} \mathrm{MZ}$ genotype) is considered disease-modifying.
With regard to the latter, $\mathrm{Pi}^{*} \mathrm{MZ}$ individuals have an increased risk of developing cirrhosis when chronic liver injury of another etiology co-exists. This has been particularly convincingly demonstrated in children with cystic fibrosis-related liver disease ${ }^{52}$ as well as in adults with NAFLD and alcoholic fatty liver disease (ALD). ${ }^{51,45}$ In all of these studies, heterozygous $\mathrm{Pi}^{*} \mathrm{Z}$ carriage conferred a strong risk for development of cirrhosis with an odds ratio as high as $5-7$. Moreover, in a large genome-wide association study, the $\mathrm{Pi}^{*} \mathrm{Z}$ variant was the single-nucleotide polymorphism conferring the strongest odds ratio to develop ALD-/NAFLD-related cirrhosis. ${ }^{51}$ Notably, the heterozygous $\mathrm{Pi}^{*} \mathrm{Z}$ presence may not only affect the risk to suffer from cirrhosis, but may also lead to faster hepatic decompensation ${ }^{53}$ and thereby result in increased morbidity and mortality. While the risk of de novo HCC development in $\mathrm{Pi}^{*} \mathrm{MZ}$ heterozygotes has not been systematically studied, ${ }^{50} \mathrm{Pi}^{*} \mathrm{MZ}$ adults with cirrhosis due to ALD or NAFLD do not seem to have an increased HCC risk compared to cirrhosis patients without $\mathrm{Pi}^{*} \mathrm{Z}$ carriage. ${ }^{51}$ As heterozygous $\mathrm{Pi}^{*} \mathrm{Z}$ carriage is quite common ( $2 \%-4 \%$ of the white population) it appears useful to screen for $\mathrm{Pi}^{*} \mathrm{Z}$ carriage in patients with chronic liver disease and inform them about their risk of rapid progression (e.g., more pronounced portal hypertension ${ }^{49}$ as well as to counsel them to avoid hepatologic risk factors (e.g., alcohol misuse, obesity, and metabolic syndrome). Such a genotyping might also be useful to prioritize them in listing for liver transplantation. ${ }^{53}$ Finally, a recent study investigating the natural history of liver disease in $\mathrm{Pi}^{*} \mathrm{MZ}$ individuals without concomitant liver disease showed that heterozygous $\mathrm{Pi}^{*} \mathrm{Z}$ carriage is not only a disease modifier of cirrhosis development, but also associated with a distinct liver phenotype. ${ }^{54}$

In conclusion, the available evidence demonstrates that $\mathrm{Pi}^{*} \mathrm{ZZ}$ homozygotes are 10-20x more likely to develop advanced liver fibrosis and that they more commonly have liver steatosis with metabolic alterations. Heterozygous $\mathrm{Pi}^{*} \mathrm{Z}$ carriage ( $\mathrm{Pi}^{*} \mathrm{MZ}$ genotype) is a strong risk factor for cirrhosis development, especially in the context of alcoholic misuse or non-alcoholic fatty liver disease. Further work is needed to determine the hepatic consequences of less prominent AAT variants including the compound heterozygous $\mathrm{Pi}^{*} \mathrm{SZ}$ genotype. 


\section{How to Approach Liver Disease in Adults with Alpha-1 Antitrypsin Deficiency?}

For the clinical routine, there are 3 major questions:

- Which AATD individuals should be assessed for the presence of liver disease?

- How should these individuals be monitored?

- How should AATD patients with signs of liver disease be managed?

While longitudinal studies systematically assessing the occurrence of liver disease in $\mathrm{Pi}^{*} \mathrm{ZZ}$ and $\mathrm{Pi}^{*} \mathrm{MZ}$ adults are missing, the current data suggests that health care providers should be aware of the relatively high risk of liver disease in these individuals and to, at least, determine serum liver enzymes regularly (i.e., alanine aminotransferase, aspartate aminotransferase, GGT, alkaline phosphatase, and bilirubin). Surprisingly, in a large multinational cohort of 554 adult $\mathrm{Pi}^{*} \mathrm{ZZ}$ homozygotes, $55 \%$ did not receive regular liver monitoring by serial measurements of liver enzymes, ${ }^{38}$ despite the fact that this surveillance can be offered easily and at low cost.

The hepatologic monitoring of $\mathrm{Pi}^{*} \mathrm{ZZ}$ adults is particularly challenging because of the striking heterogeneity of liver disease and because most $\mathrm{Pi}^{*} \mathrm{ZZ}$ individuals display normal serum liver enzymes and in spite of that, some of them may already have advanced liver fibrosis. ${ }^{38,39}$ We recommend at least an initial, non-invasive assessment of liver disease in $\mathrm{Pi}^{*} \mathrm{ZZ}$ individuals with elastography as well as with liver ultrasound. Alternatively, a non-invasive evaluation with an indirect fibrosis test can be carried out and individuals with intermediate or higher risk should be further assessed.

While there is no evidence-based hepatologic care plan for individuals with AATD-related liver fibrosis yet, based on the above mentioned findings, ${ }^{38,39}$ we recommend to approach these individuals in the same manner as patients with other liver disease entities. As detailed above, liver diseases should be evaluated by blood-based parameters, ultrasonography, and an invasive or non-invasive assessment of liver fibrosis and the management should depend on the determined amount of fibrosis. In particular, individuals with end-stage liver disease and preserved lung function constitute ideal candidates for liver transplantation ${ }^{55}$ and patients with both $\mathrm{Pi}^{*}$ ZZ-related lung and liver disease can be evaluated for combined lung and liver transplantation. Cirrhotics should be also subjected to ultrasound-based HCC surveillance every 6 months and screened for the potential presence of esophageal varices.

Individuals with less advanced liver fibrosis likely require a less stringent monitoring and the exact frequency of monitoring should be determined individually, based on the patient genotype, preferences, expected amount of fibrosis, as well as the presumed disease activity. The clinical context including age, sex, presence of comorbidities (such as obesity, diabetes mellitus, metabolic syndrome, or alcohol misuse) should also be taken into consideration. Moreover, AATD individuals should be counseled about the established risk factors and to maintain an active, healthy lifestyle, normal BMI, and to abstain from risky alcohol consumption. Individuals with recurrently elevated liver enzymes should be screened for presence of comorbidities and once these are excluded, suggested to perform liver biopsy to rule-out presence of concomitant liver disease.

As no evidence-based hepatologic care plan exists, it is recommended to refer $\mathrm{Pi}^{*} \mathrm{ZZ}$ individuals- especially those with signs of liver disease-to a health care center specializing in AATD-related liver disease. Moreover, these centers can evaluate whether an individual is suitable for one of the therapeutic trials that are currently being initiated. In our center, we recommend to control liver enzymes at least every 6-12 months and to perform an assessment of liver fibrosis every 1-2 years. Given that the risk of HCC has not been clearly determined, we tend to perform liver ultrasounds once a year in non-cirrhotic individuals. (Figure 1)

\section{Acknowledgements}

We thank the national patient organizations (i.e., Alpha-1 Deutschland: Marion Wilkens and Gabi Niethammer; Alpha-1 Austria: Ella Geiblinger; Alpha-1 Belgium: Frank Willersinn; Alpha-1 Netherlands: Heleen Groen; Alpha-1 Denmark: Gunhil Norhave, as well as Alpha-1 Spain, Alpha-1 Italy, Alpha-1 Poland, and Alpha-1 Portugal for their help with the execution of our study. We also thank all patients for their participation in our ongoing Europe-wide registry.

\section{Declaration of Interest}

PS has received speaker or consulting fees from Grifols, CSL Behring, Alnylam, and Arrowhead Pharmaceuticals. $\mathrm{KH}$ has received speaker fees from CSL Behring. 


\title{
Figure 1. Suggestion of Hepatologic Care for Adults with Alpha-1 Antitrypsin Deficiency
}

\author{
Adults with known AATD
}

Counseling (genetic advice, risk factors, family screen) Liver biochemistry (ALT, AST, GGT, ALP, bilirubin)

Liver ultrasound (signs of cirrhosis, HCC)

Blood-based liver fibrosis tests (e.g., APRI)

Liver elastography (e.g., VCTE)

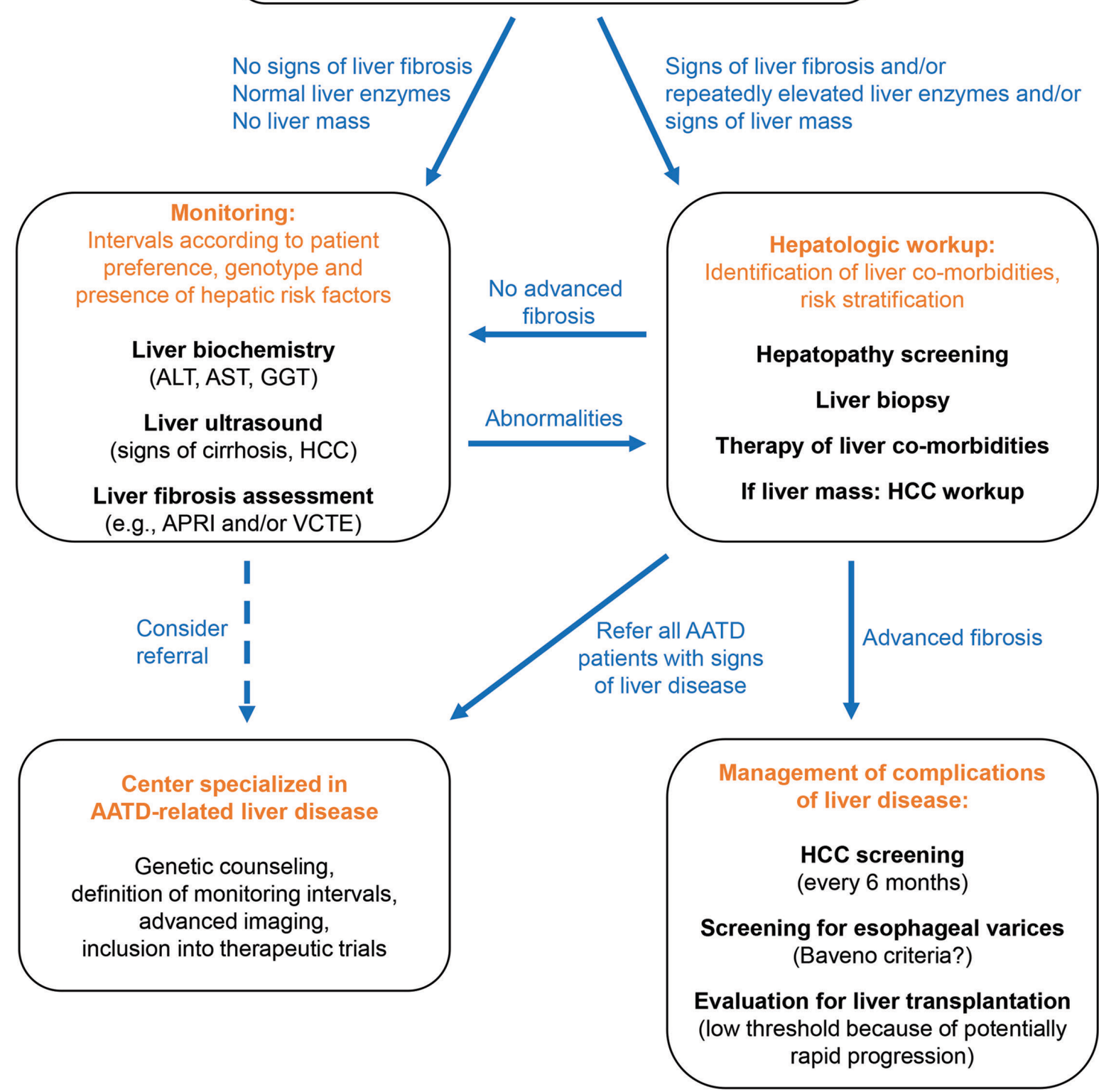

All individuals with known AATD should receive regular liver care until further evidence suggests otherwise.

AATD=alpha- 1 antitrypsin deficiency; ALT=alanine aminotransferase; AST=aspartate aminotransferase; GGT=gamma-glutamyl transferase; ALP=alkaline phosphatase; HCC=hepatocellular carcinoma; APRI=AST-to-platelet ratio index; VCTE=vibration-controlled transient elastography (FibroScan) 


\section{References}

1. 1. Ge PS, Runyon BA. Treatment of patients with cirrhosis. $N$ Engl J Med. 2016;375:767-77.

doi: https://doi.org/10.1056/NEJMra 1504367

2. Zoubek ME, Trautwein C, Strnad P. Reversal of liver fibrosis: from fiction to reality. Best Pract Res Clin Gastroenterol. 2017;31(2):129141. doi: https://doi.org/10.1016/j.bpg.2017.04.005

3. Angulo P, Kleiner DE, Dam-Larsen S, et al. Liver fibrosis, but no other histologic features, is associated with long-term outcomes of patients with nonalcoholic fatty liver disease. Gastroenterology. 2015;149(2):389-397.e10. doi: https://doi.org/10.1053/j.gastro.2015.04.043

4. Ekstedt M, Hagstrom $\mathrm{H}$, Nasr $\mathrm{P}$, et al. Fibrosis stage is the strongest predictor for disease-specific mortality in NAFLD after up to 33 years of follow-up. Hepatology. 2015;61(5):15471554. doi: https://doi.org/10.1002/hep.27368

5. Tapper EB, Lok AS. Use of liver imaging and biopsy in clinical practice. N Engl J Med. 2017;377:756-768. doi: https://doi.org/10.1056/NEJMra1610570

6. Udell JA, Wang CS, Tinmouth J, et al. Does this patient with liver disease have cirrhosis? JAMA. 2012;307(8):832-842. doi: https://doi.org/10.1001/jama.2012.186

7. Fleming KM, Aithal GP, Card TR, West J. All-cause mortality in people with cirrhosis compared with the general population: a population-based cohort study. Liver Int. 2012;32(1):79-84. doi: https://doi.org/10.1111/j.1478-3231.2011.02517.x

8. D’Amico G, Garcia-Tsao G, Pagliaro L. Natural history and prognostic indicators of survival in cirrhosis: a systematic review of 118 studies. J Hepatol. 2006;44(1):217-231. doi: https://doi.org/10.1016/j.jhep.2005.10.013

9. Villanueva A. Hepatocellular carcinoma. $N$ Engl $J$ Med. 2019;380:1450-1462.

doi: https://doi.org/10.1056/NEJMra 1713263

10. European Association for the Study of the Liver. EASL Clinical Practice Guidelines for the management of patients with decompensated cirrhosis. J Hepatol. 2018;69(2):406-460. doi: https://doi.org/10.1016/j.jhep.2018.03.024

11. Bravo AA, Sheth SG, Chopra S. Liver biopsy. N Engl J Med, 2001;344:495-500.

doi: https://doi.org/10.1056/NEJM200102153440706

12. The French METAVIR Cooperative Study Group, Bedossa P. Intraobserver and interobserver variations in liver biopsy interpretation in patients with chronic hepatitis C. Hepatology. 1994;20(1):15-20. doi: https://doi.org/10.1002/hep.1840200104

13. Regev A, Berho M, Jeffers LJ, et al. Sampling error and intraobserver variation in liver biopsy in patients with chronic HCV infection. Am J Gastroenterol. 2002;97(10):2614-2618. doi: https://doi.org/10.1111/j.1572-0241.2002.06038.x .
14. Ratziu V, Charlotte F, Heurtier A, et al. Sampling variability of liver biopsy in nonalcoholic fatty liver disease. Gastroenterology. 2005;128(7):1898-1906.

doi: https://doi.org/10.1053/j.gastro.2005.03.084

15. Colloredo G, Guido M, Sonzogni A, Leandro G. Impact of liver biopsy size on histological evaluation of chronic viral hepatitis: the smaller the sample, the milder the disease. $J$ Hepatol. 2003;39(2):239-244.

doi: https://oi.org/10.1016/S0168-8278(03)00191-0

16. Chou R, Wasson N. Blood tests to diagnose fibrosis or cirrhosis in patients with chronic hepatitis $C$ virus infection: a systematic review. Ann Intern Med. 2013;158(11):807-820.

doi: https://doi.org/10.7326/0003-4819-158-11-201306040-00005

17. Friedrich-Rust M, Poynard T, Castera L. Critical comparison of elastography methods to assess chronic liver disease. Nat Rev Gastroenterol Hepatol. 2016;13:402-411. doi: https://doi.org/10.1038/nrgastro.2016.86

18. Pereira VM, Hamesch K, Strnad P. Liver fibrosis assessment in adults with alpha-1 antitrypsin deficiency. In: Mueller S. (eds) Liver Elastography. Springer, Cham; 2020.

19. Tapper EB, Robson SC, Malik R. Coagulopathy in cirrhosis - the role of the platelet in hemostasis. J Hepatol. 2013;59(4):889-890. doi: https://doi.org/10.1016/j.jhep.2013.03.040

20. Poynard T, Vergniol J, Ngo Y, et al. Staging chronic hepatitis B into seven categories, defining inactive carriers and assessing treatment impact using a fibrosis biomarker (FibroTest ${ }^{\circledR}$ ) and elastography (FibroScan ${ }^{\circledR}$ ). J Hepatol. 2014;61(5):994-1003. doi: https://doi.org/10.1016/j.jhep.2014.06.027

21. Poynard T, Vergniol J, Ngo Y, et al. Staging chronic hepatitis $C$ in seven categories using fibrosis biomarker (FibroTest) and transient elastography (FibroScan ${ }^{\circledR}$ ). J Hepatol. 2014;60(4):706714. doi: https://doi.org/10.1016/j.jhep.2013.11.016

22. Castera L, Foucher J, Bernard PH, et al. Pitfalls of liver stiffness measurement: a 5-year prospective study of 13,369 examinations. Hepatology. 2010;51(3):828-835. doi: https://doi.org/10.1002/hep.23425

23. European Association for Study of the Liver, Asociacion Latinoamericana para el Estudio del H. EASL-ALEH clinical practice guidelines: non-invasive tests for evaluation of liver disease severity and prognosis. J Hepatol. 2015;63(1):237-264. doi: https://doi.org/10.1016/j.jhep.2015.04.006

24. Karlas T, Petroff D, Sasso M, et al. Individual patient data metaanalysis of controlled attenuation parameter (CAP) technology for assessing steatosis. J Hepatol. 2017;66(5):1022-1030. doi: https://doi.org/10.1016/j.jhep.2016.12.022

25. Imajo K, Kessoku T, Honda Y, et al. Magnetic resonance imaging more accurately classifies steatosis and fibrosis in patients with nonalcoholic fatty liver disease than transient elastography. Gastroenterology. 2016;150(3):626-637.e7. doi: https://doi.org/10.1053/j.gastro.2015.11.048 
26. Park CC, Nguyen P, Hernandez C, et al. Magnetic resonance elastography vs transient elastography in detection of fibrosis and noninvasive measurement of steatosis in patients with biopsy-proven nonalcoholic fatty liver disease. Gastroenterology. 2017;152(2):598-607.e2.

doi: https://doi.org/10.1053/j.gastro.2016.10.026

27. Karlas T, Petroff D, Wiegand J. Collaboration, not competition: the role of magnetic resonance, transient elastography, and liver biopsy in the diagnosis of nonalcoholic fatty liver disease. Gastroenterology. 2017;152(3):479-481.

doi: https://doi.org/10.1053/j.gastro.2016.12.013

28. Strnad P, McElvaney NG, Lomas DA. Alpha-1 antitrypsin deficiency. N Engl J Med. 2020;382(15):1443-1455.

doi: https://doi.org/10.1056/NEJMra1910234

29. Greene CM, Marciniak SJ, Teckman J, et al. Alpha-1 antitrypsin deficiency. Nat Rev Dis Primers. 2016;2:16051.

doi: https://doi.org/10.1038/nrdp.2016.51

30. Strnad P, Nuraldeen R, Guldiken N, et al. Broad spectrum of hepatocyte inclusions in humans, animals, and experimental models. Compr Physiol. 2013;3(4):1393-1436. doi: https://doi.org/10.1002/cphy.c120032

31. Fromme M, Oliverius M, Strnad P. DEFI-ALFA: the French key to the alpha-1 mystery? Liver Int. 2019;39(6):1019-1021.

doi: https://doi.org/10.1111/liv.14064

32. Stoller JK, Tomashefski J Jr, Crystal RG, et al. Mortality in individuals with severe deficiency of alpha-1 antitrypsin: findings from the National Heart, Lung, and Blood Institute Registry. Chest. 2005;127(4):1196-1204. doi: https://doi.org/10.1378/chest.127.4.1196

33. Tanash HA, Nilsson PM, Nilsson JA, Piitulainen E. Clinical course and prognosis of never-smokers with severe alpha-1 antitrypsin deficiency (PiZZ). Thorax. 2008;63(12):1091-1095. doi: https://doi.org/10.1136/thx.2008.095497

34. Brunt EM, Wong VW, Nobili V, et al. Nonalcoholic fatty liver disease. Nat Rev Dis Primers. 2015;1:15080. doi: https://doi.org/10.1038/nrdp.2015.80

35. Stender S, Kozlitina J, Nordestgaard BG, Tybjaerg-Hansen A, Hobbs HH, Cohen JC. Adiposity amplifies the genetic risk of fatty liver disease conferred by multiple loci. Nat Genet. 2017. doi: https://doi.org/10.1038/ng.3855

36. Turner AM, Stolk J, Bals R, et al. Hepatic-targeted RNA interference provides persistent knockdown of alpha-1 antitrypsin levels in ZZ patients. J Hepatol. 2018;69(2):378-384. doi: https://doi.org/10.1016/j.jhep.2018.03.012

37. Clark VC, Dhanasekaran R, Brantly M, Rouhani F, Schreck P, Nelson DR. Liver test results do not identify liver disease in adults with alpha (1)-antitrypsin deficiency. Clin Gastroenterol Hepatol. 2012;10(11):1278-1283. doi: https://doi.org/10.1016/j.cgh.2012.07.007
38. Hamesch K, Mandorfer M, Pereira VM, et al. Liver fibrosis and metabolic alterations in adults with alpha-1 antitrypsin deficiency caused by the PI*ZZ mutation. Gastroenterology. 2019;157(3):705-719.

doi: https://doi.org/10.1053/j.gastro.2019.05.013

39. Clark VC, Marek G, Liu C, et al. Clinical and histologic features of adults with alpha-1 antitrypsin deficiency in a non-cirrhotic cohort. J Hepatol. 2018;69(6):1357-1364. doi: https://doi.org/10.1016/j.jhep.2018.08.005

40. Kümpers J, Fromme M, Schneider CV, et al. Assessment of liver phenotype in adults with severe alpha-1 antitrypsin deficiency (Pi*ZZ genotype). J Hepatol. 2019;71(6):1272-1274. doi: https://10.1016/j.jhep.2019.08.011

41. Adam R, Karam V, Delvart V, et al. Evolution of indications and results of liver transplantation in Europe. A report from the European Liver Transplant Registry (ELTR). J Hepatol. 2012;57(3):675-688.

doi: https://doi.org/10.1016/j.jhep.2012.04.015

42. Clark VC. Liver transplantation in alpha- 1 antitrypsin deficiency. Clin Liver Dis. 2017;21(2):355-365.

doi: https://doi.org/10.1016/j.cld.2016.12.008

43. Townsend SA, Edgar RG, Ellis PR, Kantas D, Newsome PN, Turner AM. Systematic review: the natural history of alpha-1 antitrypsin deficiency, and associated liver disease. Aliment Pharmacol Ther. 2018;47(7):877-885. doi: https://doi.org/10.1111/apt.14537

44. Tanash HA, Piitulainen E. Liver disease in adults with severe alpha-1 antitrypsin deficiency. J Gastroenterol. 2019;54:541-548. doi: https://doi.org/10.1007/s00535-019-01548-y

45. Strnad P, Buch S, Hamesch K, et al. Heterozygous carriage of the alpha- 1 antitrypsin $\mathrm{Pi}^{*} \mathrm{Z}$ variant increases the risk to develop liver cirrhosis. Gut. 2019;68(6):1099-1107. doi: https://doi.org/10.1136/gutjnl-2018-316228

46. Kim RG, Nguyen P, Bettencourt R, et al. Magnetic resonance elastography identifies fibrosis in adults with alpha-1 antitrypsin deficiency liver disease: a prospective study. Aliment Pharmacol Ther. 2016;44(3):287-299. doi: https://doi.org/10.1111/apt.13691

47. Reiter R, Wetzel M, Hamesch K, et al. Comparison of noninvasive assessment of liver fibrosis in patients with alpha-1 antitrypsin deficiency using magnetic resonance elastography (MRE), acoustic radiation force impulse (ARFI) quantification, and 2D-shear wave elastography (2D-SWE). PloS One. 2018; 13:e0196486.

doi: https://doi.org/10.1371/journal.pone.0196486

48. Diaz S, Mostafavi B, Tanash HA, Piitulainen E. Acoustic radiation force impulse (ARFI) elastography in a cohort of alpha- 1 antitrypsin-deficient individuals and healthy volunteers. Acta Radiologica Open. 2018;7:2058460118768363. doi: https://doi.org/10.1177/2058460118768363 
49. Mandorfer M, Bucsics T, Hutya V, et al. Liver disease in adults with a1-antitrypsin deficiency. United European Gastroenterol J. 2018;6(5):710-718.

doi: https://doi.org/10.1177/2050640618764057

50. Eriksson S, Carlson J, Velez R. Risk of cirrhosis and primary liver cancer in alpha-1 antitrypsin deficiency. $N$ Engl J Med. 1986;314:736-739.

doi: https://doi.org/10.1056/NEJM198603203141202

51. Abul-Husn NS, Cheng X, Li AH, et al. A protein-truncating HSD17B13 variant and protection from chronic liver disease. $N$ Engl J Med. 2018;378:1096-1106. doi: https://doi.org/10.1056/NEJMoa 1712191

52. Bartlett JR, Friedman KJ, Ling SC, et al. Genetic modifiers of liver disease in cystic fibrosis. JAMA. 2009;302(10):1076-1083. doi: https://doi.org/10.1001/jama.2009.1295

53. Schaefer B, Mandorfer M, Viveiros A, et al. Heterozygosity for the alpha-1 antitrypsin Z-allele in cirrhosis is associated with more advanced disease. Liver Transpl. 2018;24(6):744-751.

doi: https://doi.org/10.1002/lt.25057

54. Schneider CV, Hamesch K, Gross A, et al. Liver phenotypes of European adults heterozygous or homozygous for $\mathrm{Pi}^{*} \mathrm{Z}$ variant of AAT ( $\mathrm{Pi}^{*} \mathrm{MZ}$ vs $\mathrm{Pi}^{*} \mathrm{ZZ}$ genotype) and non-carriers. Gastroenterology. 2020; In press.

doi: https://doi.org/10.1053/j.gastro.2020.04.058

55. Carey EJ, Iyer VN, Nelson DR, Nguyen JH, Krowka MJ. Outcomes for recipients of liver transplantation for alpha-1 antitrypsin deficiency-related cirrhosis. Liver Transpl. 2013;19(12):13701376. doi: https://doi.org/10.1002/lt.23744 